УДК 338.1

ШЕЛОМЕНЦЕВА МАРИНА ВЛАДИМИРОВНА

научный работник Смоленского филиала ФГБОУ ВО

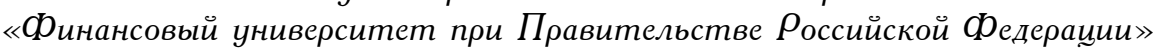

(

e-mail: mvshelomenceva@gmail.com

\title{
РАЗВИТИЕ ВЫСШЕЙ ШКОЛЫ В УСАОВИЯХ ЦИФРОВИЗАЦИИ ЭКОНОМИКИ
}

Аннотауия. Актуальность. На современном этапе необходимым условием повышения конкурентоспособности национальных инновационных экосистем является йифровизациия. Проиессы ијифровой трансформации оказывают влияние на взаимодействие субъектов инновационной деятельности в различных сферах экономики. Естественным путем все это отражается и влияет на систему высшего образования. Появляется необходимость анализа и исследования развития высшей школы в условиях илифровой эры. Џель работы: анализ эффекта влияния циировых технологий на развитие высшей школь. Метод или методология исследования: сопоставление территорий по эффекту влияния цифровых технологий на развитие высшей школы основывается на общенаучной методологии количественного и качественного анализа. В качестве информационной базы исследования использованы результаты работ российских и зарубежных специалистов, данные статистических наблюдений по индикаторам иифровой экономики, инноваций, науки, технологий. Изучение факторов, оказывающих влияние на процессы модернизации современного общества, демонстрирует важную роль илифровых технологий как драйвера, синергийно увеличивающего значимость инновационного взаимодействия государства, науки и бизнеса. Результаты. Выявлены особенности инновациинного взаимодействия субъектов национальных экономических систем. Применение методов DEA-анализа предоставило возможность получить оценки эффекта влияния циифровых технологий на

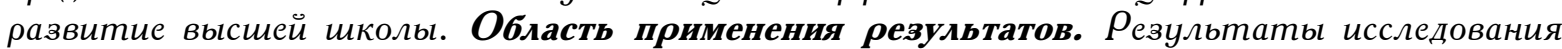
могут быть использованы органами государственной власти и органами местного самоуправления при составлении планов экономического развития отраслей региональной экономики, разработке стратегий и программ развития в области ииифровой трансформациии высшей школь. Выводы. У ровень развития национальной высшей школы рассматривается как один из ключевых показателей, которые предопределяют инноваиионный потенциал страны. Результаты исследования формируют научную основу для обоснования необходимости проведения внутренней государственной политики, ориентированной на развитие эффективного взаимодействия между субъектами инновационной деятельности в условиях ицифровизациии экономики.

Ключевые слова: инновационное развитие, эффект колеи, драйверь модернизации, внешние шоки развития, факторы развития, высшая школа, Смоленская область.

SHELOMENTSEVA MARINA VLADIMIROVNA

researcher of the Smolensk branch $O F$ the Financial University under the Government of the Russian Federation (Financial University), e-mail: mvshelomenceva@gmail.com

\section{DEVELOPMENT OF HIGHER EDUCATION IN THE CONTEXT OF DIGITALIZATION OF THE ECONOMY}

Abstract. Relevance. At the present stage, digitalization is a necessary condition for increasing the competitiveness of national innovation ecosystems. The process of digital transformation have

${ }^{1}$ Статья подготовлена при финансовой поддержке гранта РФФИ № 19-45-670001 ра «Эффект колеи: традиционное и инновационное в социально-экономическом развитии Смоленской области». 
an impact on the interaction of subjects of innovative activity in various sectors of the economy. All this naturally affects and affects the higher education system. There is a need to analyze and study the development of higher education in the digital era. The purpose of the work: to analyze the effect of digital technologies on the development of higher education. Research method or methodology: the comparison of territories by the effect of digital technologies on the development of higher education is based on the General scientific methodology of quantitative and qualitative analysis. The research is based on the results of Russian and foreign experts ' work and statistical observations on indicators of the digital economy, innovation, science, and technology. The study of factors that influence the processes of modernizing modern society demonstrates the important role of digital technologies as a driver that synergistically increases the importance of innovative interaction between the state, science and business. Results. Features of innovative interaction of subjects of national economic systems are revealed. The use of DEA analysis methods made it possible to obtain estimates of the effect of digital technologies on the development of higher education. The scope of the results. The results of the research can be used by state authorities and local self-government bodies in drawing up plans for the economic development of regional economic sectors, developing strategies and development programs in the field of digital transformation of higher education. Conclusions. The level of development of the national higher school is considered as one of the key indicators that determine the innovative potential of the country. The results of the study form the scientific basis for justifying the need for an internal state policy focused on the development of effective interaction between the subjects of innovative activity in the conditions of digitalization of the economy.

Keywords: innovative development, track effect, modernization drivers, external development shocks, development factors, higher school, Smolensk region.

Введение. Для современного общества характерна высокая скорость изменения конъюнктуры рынков товаров и услуг в различных сферах экономики. На фоне общего курса перехода на более эффективную траекторию развития, формирующуюся под воздействием различных факторов (технологических, демографических, социальных, культурологических и др.), возрастает роль высшей школы как источника инноваций.

Создание благоприятной среды для коммерциализации результатов научноисследовательской и опытно-конструкторской работы как элемента инновационной экосистемы способствует повышению конкурентоспособности и имиджевому позиционированию национальных экономических систем [9-11].

В этой связи совершенствование методологии изучения взаимосвязи и взаимозависимости, возникающих в процессе функционирования и взаимодействия различных субъектов инновационной экосистемы, рассматривается как перспективное направление научных исследований на современном этапе.

Проблема исследования. В конце XX века были опубликованы результаты первыХ исследований по изучению QWERTY-эффектов, которые обратили внимание научного сообщества на институциональные изменения и на роль институтов в научно-техническом прогрессе [1214]. Данная теория получила развитие в работах лауреата Нобелевской премии Д. Норта (1993), который выдвинул концепцию «Path Dependency» (в научной литературе наиболее известна как «зависимость от ранее выбранного пути», «эффект колеи»). Под этим термином он имеет в виду «последствия небольших событий и случайных обстоятельств, определяющих решения, которые, будучи приняты, направляют развитие по определенному пути» [15, p. 92].

Изучение гипотезы Норта - Дэвида представителями российской экономической школы (Полтеровича В. М., Аузана А. А., Нуреева Р. М. и др.) позволило выявить тот факт, что наблюдающийся «эффект колеи» в основном обусловлен «институциональными ловушками» российской экономики и социально-культурными аспектами трансформации экономической структуры России.

Результаты проведенных исследований зарубежных и отечественных ученых, посвященных «Path Dependency», позволяют сделать следующие выводы. Во-первых, проявления теории зависимости от предшествующего развития в экономической истории институтов изучаются, как правило, или на уровне отдельных институтов (правовых, организационных, политических и т. д.), или на уровне национальных экономических систем. 
Во-вторых, многообразие взаимосвязей и взаимовлияний между физическим капиталом (системы оборудования) и человеческим капиталом (навыки работников) формируется под воздействием множества факторов: технологических, социальных, культорологических и др.

Эволюция подходов к исследованию «эффекта колеи» показывает, что если на ранних этапах исследователями выделялись технологические факторы, то в качестве современных причин рассматриваются культурологические факторы: общественное согласие, ментальность, образование и другие [16-18]. Сближение институциональной экономической истории с эволюционной экономической теорией проявляется в том, что институты как факторы стандартизации и унификации определяют выбор траектории развития, а «устойчивой колеей» ее делает культура.

Переход от экспортно-сырьевой модели развития экономики к инновационной модели, основанной на эффективном использовании экономических ресурсов за счет применения высокопроизводительных наукоемких технологий (high-tech), оказывает существенное влияние на процессы модернизации современного общества.

В условиях экономической неопределенности, обусловленной современными социальнодемографическими «вызовами» и последствиями пандемии COVID-19, актуальным направлением научных исследований становится изучение влияния цифровых технологий (технологический фактор) на развитие науки (культорологический фактор). Полученные результаты формируют научную основу для обоснования необходимости проведения внутренней государственной политики, ориентированной на развитие эффективного взаимодействия между субъектами инновационной деятельности в условиях цифровизации экономики.

Методы исследования. Ключевым направлением развития современного общества является создание благоприятной среды для достижения целей $\mathrm{OOH}$ в области устойчивого развития на основе использования экологически чистых («зеленых») и цифровых технологий. В рамках данного тренда многие страны делают акцент на взаимодействии институтов и участников инновационной деятельности путем эффективной адаптации передового международного опыта по поддержке и развитию инноваций с участием государства к национальным особенностям окружающей среды (культуры, ресурсов, технологий и др.).

Анализ результатов научных источников в области устойчивого развития позволяет сделать вывод о том, что среди необходимых условий поступательного движения к экономической модели роста выделяется совершенствование механизмов инновационной коллаборации государства, предпринимательства и науки. Развитие механизма формирования инновационной коллаборации способствует обеспечению роста занятости, экспорта и инвестиций; повышению конкурентоспособности хозяйствующих субъектов на макро- и микроуровнях на основе создания и применения новых знаний и высокопроизводительных наукоемких технологий.

По мнению экспертов Всемирного банка, в процессе развития постиндустриального общества на формирование цепочек создания стоимости и продвижения инноваций в различные секторы национальных экономик наибольшее влияние оказывают составляющие инновационной инфраструктуры (производственно-технологическая, консалтинговая, финансовая, кадровая, информационная и др.), а также институциональные факторы, обеспечивающие государственную поддержку научно-исследовательской и опытно-конструкторской работы. Среди факторов, оказывающих влияние на скорость распространения инноваций, рассматривают катализаторы и барьеры инновационной деятельности. К первой группе факторов относят нематериальные активы (новые технологии, знания, торговые марки, гудвиллы и др.), государственную поддержку инноваций, льготное налогообложение и др., ко второй - слабость материально-технической базы, патентно-лицензионные ограничения в области инноваций и др.

Данные международных сопоставлений количественных показателей, характеризующих научный и инновационный потенциал, сведения об интеллектуальной собственности, результативность исследований и разработок и др. позволяют сделать вывод о качественном состоянии взаимодействия субъектов инновационной деятельности как фактора развития национальной экосистемы.

Для оценки влияния цифровых технологий (технологический фактор) на развитие высшей школы (культурологический фактор) проведен сравнительный анализ с применением идей непараметрического метода «оболочечного» анализа (Data Envelopment Analysis, DEA) [19-21] применительно к рассматриваемой совокупности единиц (Decision Making Units, DMU). 
В данном исследовании выборка единиц рассматриваемой совокупности представлена следующими странами: США, Германия, Франция (страны с развитой экономикой), Китай, Россия (страны с развивающейся экономикой и формирующимися рынками).

Ориентация на модель устойчивого экономического роста предполагает построение модели вида CCR-O, ориентированной на «результат» / «выход» (output-oriented):

DMU $U_{j}$ input: $\left(x_{1 j}, x_{2 j}, \ldots, x_{m j}\right)^{T}$,output: $\left(y_{1 j}, y_{2 j}, \ldots, y_{s j}\right)^{T}$,

где $\mathrm{m}$ - количество входных параметров $(\mathrm{i}=1, \ldots, \mathrm{m})$,

$\mathrm{s}$ - количество выходных параметров $(\mathrm{j}=1, \ldots, \mathrm{n})$,

$\mathrm{n}$ - количество DMU, (n=s+n),

$\mathrm{X}$ - матрица входных параметров

Y - матрица выходных параметров

$$
\begin{aligned}
& \left(\begin{array}{cccc}
x_{11} x_{12} & \ldots & x_{1 \mathrm{n}} \\
x_{21} & x_{22} & \ldots & x_{2 \mathrm{n}} \\
\ldots & \ldots & \ldots & \ldots \\
x_{\mathrm{m} 1} & x_{\mathrm{m} 2} & \ldots & x_{\mathrm{mn}}
\end{array}\right), \\
& \left(\begin{array}{cccc}
y_{11} & y_{12} & \ldots & y_{1 \mathrm{n}} \\
y_{21} & y_{22} & \ldots & y_{2 \mathrm{n}} \\
\ldots & \ldots & \ldots & \ldots \\
y_{\mathrm{s} 1} & y_{\mathrm{s} 2} & \ldots & y_{\mathrm{sn}}
\end{array}\right) .
\end{aligned}
$$

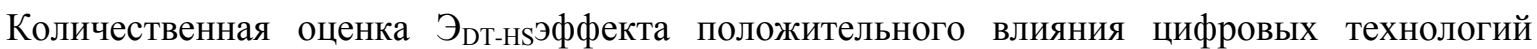
(DigitalTechnology, DT) на развитие высшей школы (Higher School, HS) определяется следующим образом:

$$
\exists_{D T-H S}=\frac{Y_{T_{H S}}}{x_{f_{\mathrm{DT}}}}
$$

Значения показателя ${ }^{\exists_{D T-H S_{f}}}(0 \leq \underbrace{\exists_{D T-H S_{f}}} \leq 1)$ отражают отношение достигнутых показателей единицы $\mathrm{DMU}_{\mathrm{j}}$ рассматриваемой совокупности к потенциально возможным. Для построения эталонного вектора результатов взаимодействия цифровых технологий и высшей школы из рассматриваемой совокупности единиц отбираются объекты $\mathrm{DMU}_{\mathrm{j}}$, чьи высокие значения показателя ${ }_{v i r-\mu s_{j}}$ используются чаще других. Такие объекты классифицируются как лидеры, в противном же случае маркируются как аутсайдеры.

Универсальность количественной оценки эффекта влияния цифровых технологий на уровень развития системы образования, в том числе высшей школы, заключается в возможности применения механизма ее построения применительно к объекту исследования при проведении международных сопоставлений, а также сравнительного анализа субъектов РФ.

Входные параметры представлены индикаторами технологического фактора, среди которых международный индекс цифровой экономики и общества (Digital Economy and Society Index; DESI) [14]; численность исследователей в расчете на 10 тыс. занятых, чел.(Res) [15]; международный рейтинг стран и территорий мира по числу регистрируемым патентов (World IntellectualPropertyIndicators, WIPI) [3]. Два последних показателя рассматриваются как индикаторы научного и интеллектуального потенциала национальной инновационной экосистемы.

Следует отметить, что выбор показателя, характеризующего патентную деятельность, обусловлен тем фактом, что уровень патентной активности может рассматриваться, с одной стороны, как индикатор коммерциализации результатов научно-исследовательской и опытноконструкторской работы, с другой - как индикатор повышения конкурентоспособности национальной инновационной экосистемы. Общепризнано, что патентная статистика является надежным показателем инновационной деятельности, и для оценки эффективности взаимодействия элементов инновационной экосистемы использование таких статистических данных является обычной мировой практикой. 
В качестве выходного параметра рассматривается интегральный показатель рейтинга национальных систем высшего образования (U21Ranking ofNationalHigherEducationSystems). Ежегодные исследования, предоставляющие возможность оценить уровень развития высшей школы в странах мира, проводятся с 2012 года. Глобальный проект международной сети университетов Universitas 21(U21) объединяет университеты из 17 стран мира (Великобритания, Китай, США, Россия, Германия, Франция и др.) и охватывает более 1,3 миллиона обучающихся и 220 тысяч преподавателей [4].

Информационная база исследования представлена данными нижеследующей таблицы, где значения входных и выходных параметров по рассматриваемой совокупности стран на начало 2019 года размещены в графах 1-4, результаты расчетов с применением технологий DEAанализа - в графах 5-7.

Таблица 1

Распределение стран по индикаторам науки, инноваций, технологий

\begin{tabular}{|l|c|c|c|c|c|c|c|}
\hline \multirow{2}{*}{ DMU } & \multicolumn{2}{|c|}{$\begin{array}{c}\text { Входные параметры } \\
\text { (input) }\end{array}$} & $\begin{array}{c}\text { Выходной } \\
\text { параметр } \\
\text { (output) }\end{array}$ & \multicolumn{3}{|c|}{ Результаты } \\
\cline { 2 - 8 } & DESI & WIPI & Res & U21 & $\begin{array}{c}\text { Показатель эф- } \\
\text { фекта влияния } \\
\text { Эрт-нS }\end{array}$ & $\begin{array}{c}\text { Эталонный регион } \\
\text { (коэффициент, с которым он } \\
\text { формирует гипотетический } \\
\text { объект) }\end{array}$ & $\begin{array}{c}\text { Место } \\
\text { (ранг) }\end{array}$ \\
\hline A & 1 & 2 & 3 & 4 & 5 & 6 & 7 \\
\hline Германия & 0,64 & 6,00 & 95,00 & 69,60 & 0,72 & США (0,95) & 4 \\
\hline Китай & 0,45 & 1,00 & 22,00 & 54,70 & 1,00 & Китай $(1,00)$ & 1 \\
\hline Россия & 0,48 & 8,00 & 57,00 & 48,50 & 0,69 & Китай $(0,17) ;$ США (0,59) & 5 \\
\hline США & 0,67 & 2,00 & 89,00 & 100,00 & 1,00 & США (1,00) & 1 \\
\hline Франция & 0,62 & 15,00 & 103,00 & 67,60 & 0,73 & США (0,92) & 3 \\
\hline
\end{tabular}

Источник: составлено автором.

Для обработки информационной базы исследования выбран программный продукт MaxDEA Software (http://maxdea.com), предоставляющий возможность импортировать входные данные, подготовленные средствами EXCEL, и экспортировать результаты DEA-анализа в виде EXCEL -таблиц.

Результаты. Международные сопоставления на основе количественного показателя оценки эффекта влияния цифровых технологий на развитие высшей школы позволяют сделать следующие выводы. Наиболее существенный положительный эффект наблюдается в Китае и США, показатели которых являются ориентиром для остальных стран. Следует отметить, что для США одновременно характерны высокая патентная активность и высокие значения индекса цифровизации экономики.

Для России эталонное множество включает показатели Китая и США. Поскольку весовой коэффициент для США (вес 0,596) больше, чем для Китая (вес 0,179), то это значит, что структура значений показателей России ближе к структуре показателей США.

Для осуществления «инновационного» рывка, способствующего вхождению российской системы высшего образования в число ведущих университетов стран мира, России необходимо решать задачи не только преодоления технологического цифрового разрыва, но и осуществления цифровой трансформации образования, включая высшую школу [6-8].

Качественное решение задачи по преодолению эффекта зависимости от предшествующего пути развития предполагается по результатам реализации национальных проектов на территории субъектов Российской Федерации. Так, национальный проект «Образование» направлен на обновление содержания российской системы образования, включая и высшую школу, создание необходимой современной инфраструктуры, подготовку соответствующих профессиональных кадров, их переподготовку и повышение квалификации, а также создание наиболее эффективных механизмов управления этой сферой.

В целях удовлетворения запросов населения на образовательные услуги, обеспечения доступности к качественному образованию усиливается процесс дифференциации сети образова- 
тельных учреждений.

По результатам мониторинга эффективности деятельности образовательных организаций в отношении филиалов, создающих финансовые и репутационные риски, связанные с некачественным экспортом образовательных, научных и кадровых технологий, головными вузами принимаются решения о реорганизации или ликвидации таких филиалов. Так, за последние пять лет произошла реструктуризация образовательных учреждений в виде оптимизации филиальной сети организаций высшего образования на территории Смоленской области. Динамика оптимизации филиальной сети учреждений высшего образования на территории Смоленской области за последние пять лет представлена на нижеследующем рисунке.

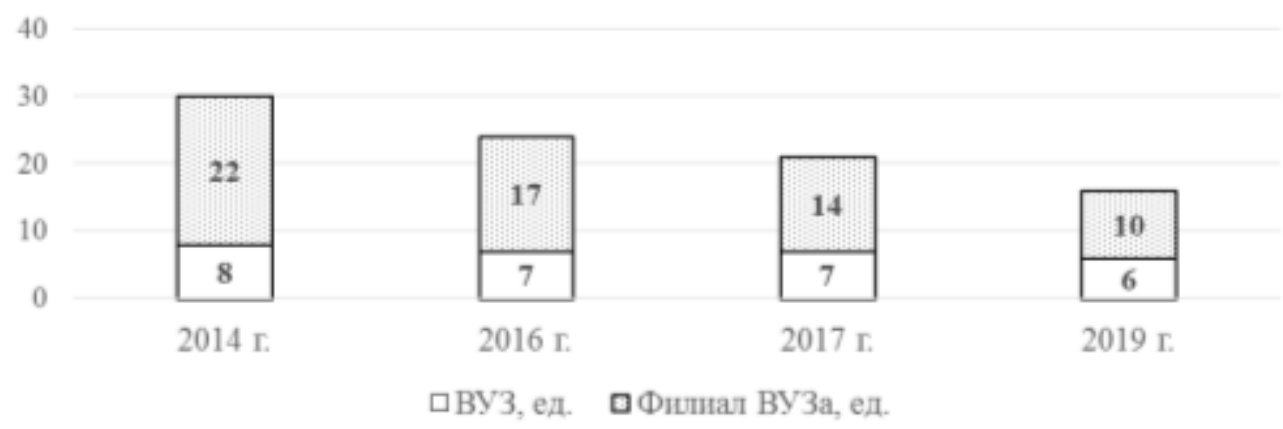

Pис. 1. Динамика оптимизации филиальной сети учреждений выстего образования на территории Смоленской области за 2014-2019 годы.

Источник: составлено автором по данным [5].

Как видно из рисунка, если на начало 2014 года на территории региона осуществляли образовательную деятельность 22 филиала высшей школы, то на начало 2020 года - 10 филиалов. Количественные показатели и качественные характеристики современной системы высшего образования Смоленской области приведены на рисунке 2.

\section{Центральный федеральный округ Смоленская область}

\section{ХАРАКТЕРИСТИКА СИСТЕМЫ ВЫСШЕГО ОБРАЗОВАНИЯ}

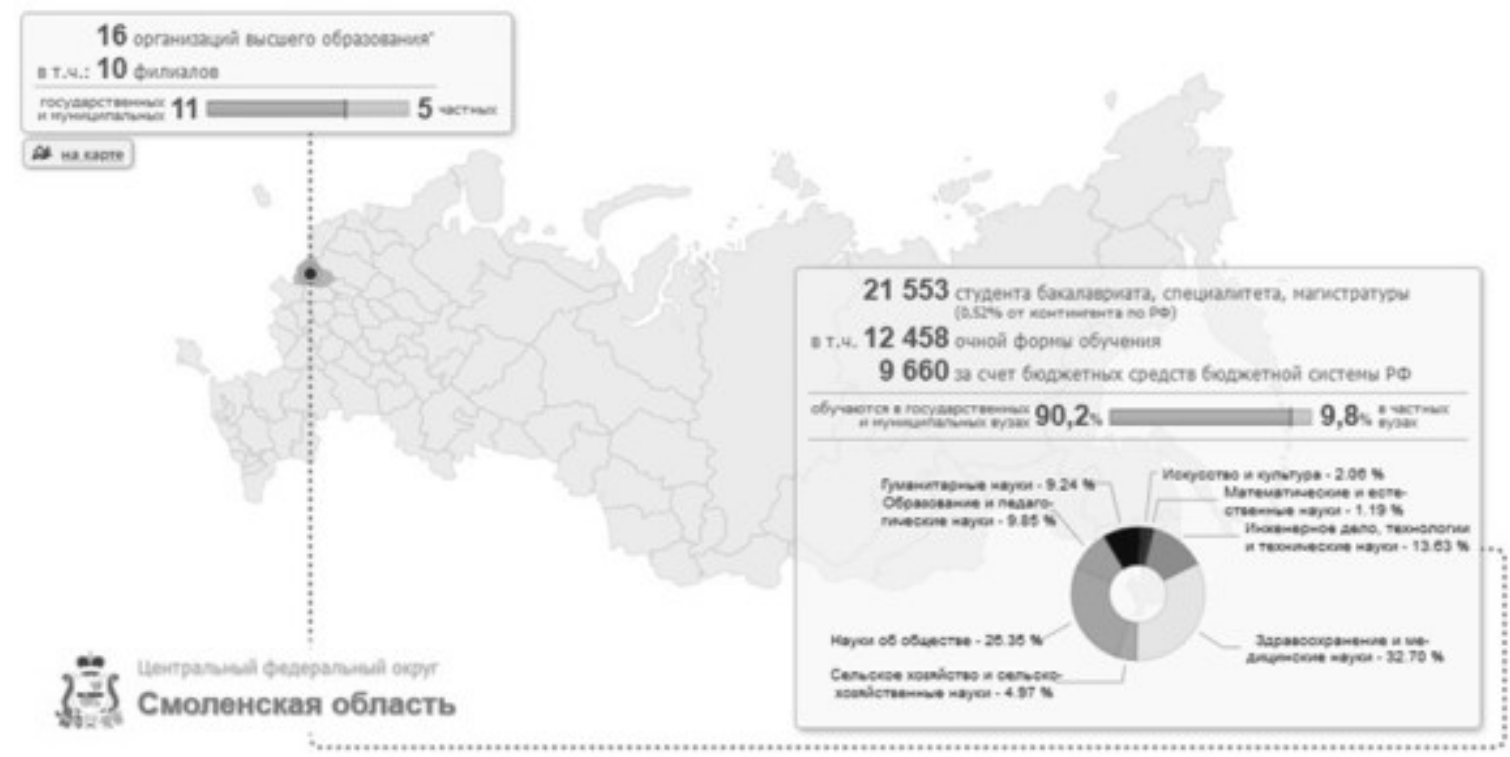

Pис. 2. Характеристика системы высшего образования Смоленской области по результатам мониторинга 2019 года.

Источник: составлено автором по данным [5]. 
Необходимым условием качественного решения задачи подготовки высококвалифицированных кадров для экономики Смоленской области является использование цифровых технологий (блокчейн, искусственный интеллект, виртуальная реальность и др.) в образовательном процессе региональной высшей школы. Цифровые технологии нацелены на повышение роли самостоятельной работы студентов, обуславливающей дальнейшее уменьшение традиционной аудиторной нагрузки студента за счет увеличения исследовательской и проектной деятельности, эффективного использования информационных и коммуникационных технологий.

Выводы. Цифровая трансформация экономики, ориентированная на эффективное и гибкое применение новейших технологий (машинное обучение, искусственный интеллект и др.), кардинально меняет рынок труда, предъявляя новые требования к системе подготовки кадров как ключевому фактору развития инновационной деятельности. Уровень развития национальной высшей школы рассматривается как один из ключевых показателей, которые предопределяют инновационный потенциал страны.

Международные сопоставления стран по индикаторам, характеризующим интенсивность и динамику развития науки, технологий, инноваций, позволяют оценить место России не только среди стран с развитой экономикой, но и среди стран, чьи показатели эффективности функционирования национальных инновационных систем сопоставимы с российскими.

К ключевым факторам, способствующим обеспечению высоких темпов долгосрочного экономического роста и повышению эффективности производства, относятся цифровые технологии (технологический фактор) и высшая школа (культурологический фактор). Именно решение задач по реализации научно-исследовательских проектов, увеличению инвестиций в научно-исследовательские и опытно-конструкторские работы, цифровизации экономики и другие, будет способствовать совершенствованию взаимодействия субъектов национальной инновационной экосистемы.

На современном рынке труда наиболее востребованы специалисты, владеющие цифровыми технологиями. Широкое внедрение цифровых технологий способствует формированию и развитию качественной информационно-образовательной среды, неотъемлемой частью которой являются:

- повышение уровня формирования профессиональных компетенций будущих специалистов;

- профессиональная переподготовка и повышение квалификации педагогических работников образовательных организаций в соответствии с современными стандартами;

- расширение введения в образовательных организациях высшего образования практики для студентов в компаниях отрасли информационных технологий и стимулирование таких компаний к открытию кафедр в образовательных организациях высшего образования;

- развитие в образовательных организациях высшего образования факультетов информационных технологий высокого уровня подготовки;

- развитие центров профессиональной переподготовки специалистов смежных областей и центров повышения квалификации молодых специалистов в сфере информационных технологий;

- введение в образовательные программы технических специальностей дисциплин, позволяющих сформировать навыки в сфере информационных технологий;

- усиление подготовки высококвалифицированных кадров, необходимых для развития наиболее перспективных критических технологий в области информационных технологий;

- привлечение бизнеса для отбора абитуриентов, для консультаций по составлению учебных программ и для прохождения стажировок.

Активизация взаимодействия всех участников инновационной экосистемы, включая государство, науку, образование и бизнес, является необходимым условием перехода к устойчивой экономической модели развития.

Литература

1. Индикаторы инновационной деятельности. 2019. Статистический сборник/ Л. М. Гохберг, К. А. Дитковский, И. А. Кузнещова и др.; Наи. исслед. ун-т «Высшая школа экономики». - М.: НИУ ВШЭ, 2019. $-376 c$.

2. Киященко Л. Т. Оценки эффективности инновационной деятельности: международный опыт и рос- 
сийская практика // Региональные проблемы преобразования экономики. - 2019. - № 7 (105). - C. 12-20. 3. Клейнер Г. Б. Экономика экосистем: шаг в будущее // Экономическое возрождение России. - 2019. № 1 (59). - C. 40-45.

4. Кондрашов В. М. Человеческий капитал и ичифровая экономика: региональный аспект // Региональные проблемы преобразования экономики. 2017. - № 12 (86). - С. 77-82.

5. Межевич H. М. К вопросу о верификации концееции «Path Dependence» для постсоветского пространства в свете тридцати лет интуитивных практик // Управленческое консультирование. 2020. - № 5. - C. 95-101. [Электронныий ресурс]. Режим доступа: DOI 10.22394/1726-1139-2020-5-95101, свободный. - Загл. с экрана.

6. Миролюбова Т. В., Карлина Т. В., Николаев Р. С. Цифровая экономика: проблемы идентификации и измерений в региональной экономике // Экономика региона. - 2020. - T.16, вып. 2. - C. 377-390. [Электронный ресурс]. Режим доступа: http://doi.org/10.17059/2020-2-4, свободный. - Загл. с экрана. 7. Мониторинг эффективности результатов деятельности организаций высшего образования: данные за 2014-2019 годы. [Электронный ресурс]. Режим доступа: http://indicators.miccedu.ru, свободный. Загл. с экрана.

8. Соловьева T. С. Теоретические аспекты формирования и развития региональных социальноинноваиионных экосистем // Вестник НГИЭИ. - 2019. - №3 (94). - С. 84-93.

9. Arthur W. Brian (1990). Positive Feedbacks in the Economy // Scientifi c American. Vol. 262, February. C. 92-99.

10. David, Paul A. (1985). Clio and the Economics of QWERTY // American Economic Review. Vol. 75. No. 2.

11. Devigne D., Vanacker T., Manigart S. and Paeleman I. (2013). The role of domestic and cross-border venture capital investors in the growth of portfolio companies. Small Business Economics, 40(3). P. 553-573.

12. [Электронный ресурс]. Режим доступа: https://digital-agenda-data.eu/, свободный. - Загл. с экрана. Наука. Технологии. Инновачии: 2020: краткий статистический сборник / Л. М. Гохберг, К. А. Дитковский, Е. И. Евневич и др.; Наи. исслед. ун-т «Высшая школа экономики». - М.: НИУ ВШЭ, 2020. - 88 c.

13. I-DESI 2018: How digital is Europe compared to other major world economies? [Электронный ресурс]. Режим доступа: https//es.europe.eu/digital-single-market/en/news/how-digital-europe-compared-othermajor-world-economies. - Date of access: 20.02.2019, свободный. - Загл. с экрана.

14. Liebowitz SJ. and Margolis S. E. (2000). Winners, Losers and Microsoft. Oakland: The IndependentInstitute.

15. Moore J. F. The death of competition: Leadership and strategy in the age of business ecosystems. 1996. New-York, NY: HarperCollins. 297 p.

16. Nahata R., Hazarika S. and Tandon K. (2014). Success in global venture capital investing: Do institutional and cultural differences matter? Journal of Financial and Quantitative Analysis, 49(4), 1039-1070Hillier D., Grinblatt M., Titman S. Financial Markets and Corporate Policy. Mc Graw Hill; 2nd edition 2012 Chapter 5, 6.

17. Nelson R. National Innovation Systems. A comparative Analysis. N.Y. Oxford: Oxford University Press 1993.

18. North D. N. Institutions, Institutional Change and Economic Performance. Cambridge University Press, 1992.

19. U21 Ranking of National Higher Education Systems. [Электронный ресурс]. Режим доступа: https:// universitas21.com/sites/default/files/2020-04/U21_Rankings\%20Report_0320_Final_LR\%20Single.pdf, c8oбодный. - Загл. с экрана.

20. Wright M., Lockett A. and Pruthi S. (2002). Internationalization of western venture capitalists into emerging markets: Risk assessment and information in India. Small Business Economics, 19(1), 13-29.

21. Zemlyak S.V. Contemporary models of government-backed venture project funding // Proceedings of the External Challenges and Risks for Russia in the Context of the World Community's Transition to Polycentrism: Economics, Finance and Business (ICEFB 2019) Cep. "Advances in Economics, Business and Management Research». 2019. P. 150-153.

\section{References:}

1. Indikatory innovacionnoj deyatel'nosti. 2019. Statisticheskij sbornik/L. M. Gohberg, K. A. Ditkovskij, I. A. Kuznecova i dr.; Nac. issled. un-t «Vysshaya shkola ekonomiki». - M.: NIU VSHE, 2019. - 376 s.

2. Kiyashchenko L. T. Ocenki effektivnosti innovacionnoj deyatel'nosti: mezhdunarodnyj opyt i rossijskaya praktika // Regional'nye problemy preobrazovaniya ekonomiki. - 2019. - № 7 (105). - S. 12-20.

3. Klejner G. B. Ekonomika ekosistem: shag v budushchee // Ekonomicheskoe vozrozhdenie Rossii. - 2019. № 1 (59). $-S$. 40-45.

4. Kondrashov V. M. CHelovecheskij kapital i cifrovaya ekonomika: regional'nyj aspekt // Regional'nye problemy preobrazovaniya ekonomiki. 2017. - № 12 (86). - S. 77-82.

5. Mezhevich N. M. K voprosu o verifikacii koncepcii «Path Dependence» dlya postsovetskogo prostranstva v svete tridcati let intuitivnyh praktik // Upravlencheskoe konsul'tirovanie. - 2020. - № 5. - S. 95-101. [Elektronnyj resurs]. Rezhim dostupa: DOI 10.22394/1726-1139-2020-5-95-101, svobodnyj. - Zagl. s ekrana. 6. Mirolyubova T. V., Karlina T. V., Nikolaev R. S. Cifrovaya ekonomika: problemy identifikacii i izmerenij v regional'noj ekonomike // Ekonomika regiona. - 2020. - T.16, vyp. 2. - S. 377-390. [Elektronnyj resurs]. Rezhim dostupa: http://doi.org/10.17059/2020-2-4, svobodnyj. - Zagl. s ekrana.

7. Monitoring effektivnosti rezul'tatov deyatel'nosti organizacij vysshego obrazovaniya: dannye za 2014-2019 gody. [Elektronnyj resurs]. Rezhim dostupa: http://indicators.miccedu.ru, svobodnyj. - Zagl. s ekrana.

8. Solov'eva T. S. Teoreticheskie aspekty formirovaniya i razvitiya regional'nyh social'no-innovacionnyh ekosistem // Vestnik NGIEI. - 2019. - №3 (94). - S. 84-93.

9. Arthur W. Brian (1990). Positive Feedbacks in the Economy // Scientifi c American. Vol. 262, February. S. 
92-99.

10. David, Paul A. (1985). Clio and the Economics of QWERTY // American Economic Review. Vol. 75. No. 2.

11. Devigne D., Vanacker T., Manigart S. and Paeleman I. (2013). The role of domestic and cross-border venture capital investors in the growth of portfolio companies. Small Business Economics, 40(3). P. 553-573.

12. [Elektronnyj resurs]. Rezhim dostupa: https://digital-agenda-data.eu/, svobodnyj. - Zagl. s ekrana. Nauka. Tekhnologii. Innovacii: 2020: kratkij statisticheskij sbornik/L. M. Gohberg, K. A. Ditkovskij, E. I. Evnevich i $d r . ;$ Nac. issled. un-t «Vysshaya shkola ekonomiki». - M.: NIU VSHE, 2020. - 88 s.

13. I-DESI 2018: How digital is Europe compared to other major world economies? [Elektronnyj resurs]. Rezhim dostupa: https//es.europe.eu/digital-single-market/en/news/how-digital-europe-compared-other-majorworld-economies. - Date of access: 20.02.2019, svobodnyj. - Zagl. s ekrana.

14. Liebowitz SJ. and Margolis S. E. (2000). Winners, Losers and Microsoft. Oakland: The IndependentInstitute.

15. Moore J. F. The death of competition: Leadership and strategy in the age of business ecosystems. 1996. New-York, NY: HarperCollins. 297 p.

16. Nahata R., Hazarika S. and Tandon K. (2014). Success in global venture capital investing: Do institutional and cultural differences matter? Journal of Financial and Quantitative Analysis, 49(4), 1039-1070Hillier D. Grinblatt M., Titman S. Financial Markets and Corporate Policy. Mc Graw Hill; 2nd edition 2012 Chapter 5, 6.

17. Nelson R. National Innovation Systems. A comparative Analysis. N.Y. Oxford: Oxford University Press 1993.

18. North D. N. Institutions, Institutional Change and Economic Performance. Cambridge University Press, 1992.

19. U21 Ranking of National Higher Education Systems. [Elektronnyj resurs]. Rezhim dostupa: https:// universitas21.com/sites/default/files/2020-04/U21 Rankings\%20Report 0320 Final LR\%20Single.pdf, Svobodnyj. - Zagl. s ekrana.

20. Wright M., Lockett A. and Pruthi S. (2002). Internationalization of western venture capitalists into emerging markets: Risk assessment and information in India. Small Business Economics, 19(1), 13-29.

21. Zemlyak S.V. Contemporary models of government-backed venture project funding // Proceedings of the External Challenges and Risks for Russia in the Context of the World Community's Transition to Polycentrism: Economics, Finance and Business (ICEFB 2019) Ser. "Advances in Economics, Business and Management Research». 2019. P. 150-153. 